\title{
Highlights of the Year
}

\author{
Physics picks its favorite stories from 2021.
}

W ith the COVID-19 pandemic still raging, life, for many, is still in limbo. But if this year's results from physics are anything to go by, physicists appear to be making the most of these difficult circumstances, with research in all fields delivering surprise findings, breakthrough results, and fun revelations. As 2021 draws to a close, Physics looks back at its favorite stories of the year.

Wishing everyone a safe and bright 2022.

-The Editors

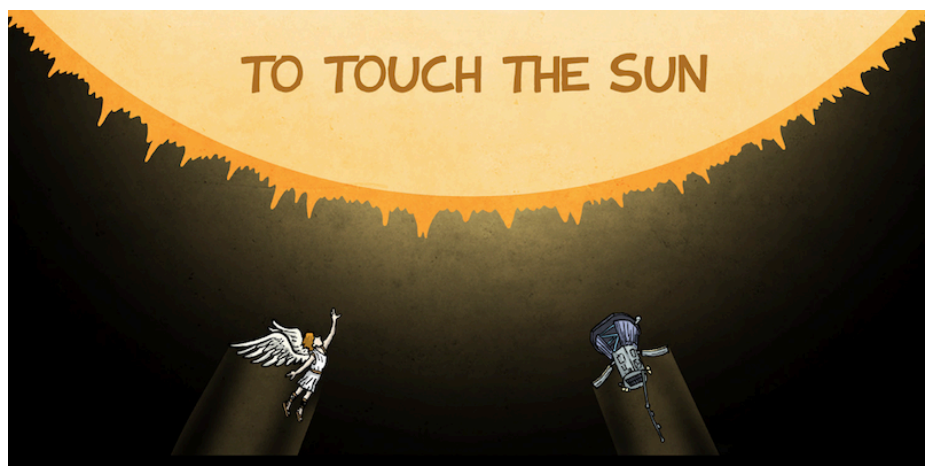

Credit: APS/Jorge Cham

\section{A Solar Rendezvous}

NASA's Parker Solar Probe has gone closer to the Sun than any other human-made object. In April of this year, the spacecraft's trajectory brought it within 18 solar radii (13 million kilometers) of the Sun's center. There, it entered a highly magnetized region of the solar corona, where magnetic energy dominates the kinetic energy of the plasma (see our special coverage: Viewpoint: Momentous Crossing of a Solar Boundary, Special Feature: To Touch the Sun, Research News: How to Survive Flying Too Close to the Sun, and Q\&A: Sun-Struck Scientist). The probe measured the plasma turbulence and magnetic-field fluctuations, providing solar scientists with highly desired data

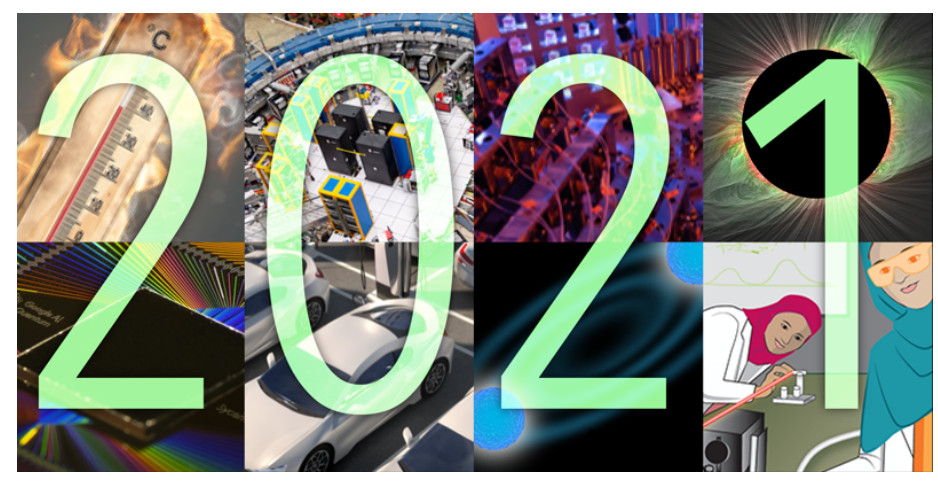

Credit: APS/Alan Stonebraker

on the mechanisms that drive the solar wind. The observations may also shed light on why the corona is a thousand times hotter than the Sun's surface.

\section{Muon Strengthens Its Challenge to the Standard Model}

The most highly anticipated particle physics result came from the Muon $g-2$ Experiment at Fermi National Accelerator Laboratory: The collaboration reported a new measurement of the magnetism of a muon, the electron's heavier cousin (see Viewpoint: Muon's Escalating Challenge to the Standard Model). Since 2001, measurements have suggested that the muon is slightly more magnetic than theory predicts. The mismatch is intriguing because it could be caused by particles or by interactions that aren't considered in the standard model of particle physics. Physics teamed up with Jorge Cham, aka PHD Comics, to illustrate the meaning of the $g-2$ anomaly in a cartoon (see Special Feature: The Muon g-2 Anomaly Explained).

\section{Entering the Age of Quantum Primacy?}

Quantum computing is a fast-moving field, and this year, the title of "largest quantum computer" shifted from Google to the University of Science and Technology of China (USTC) to IBM in less than a month. The USTC results stand out because they 


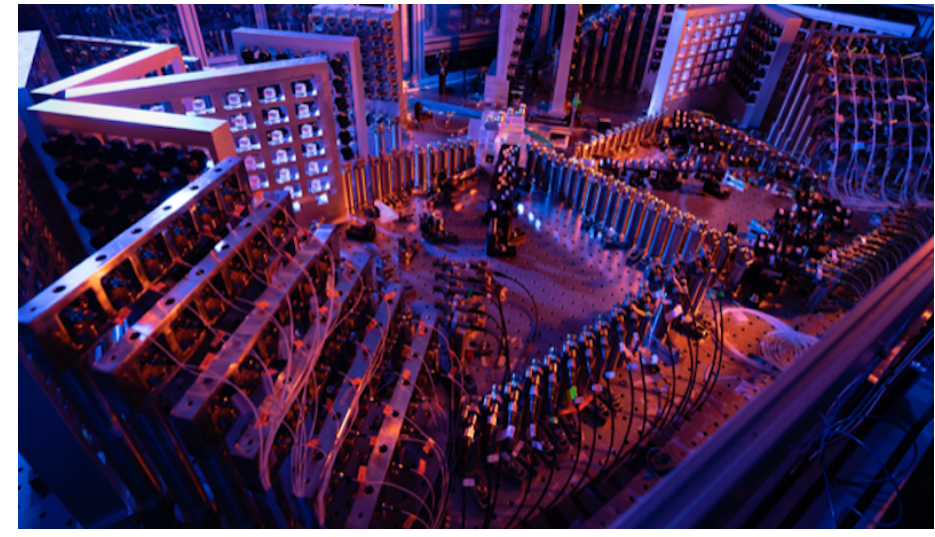

Credit: Chao-Yang Lu/University of Science and Technology of China

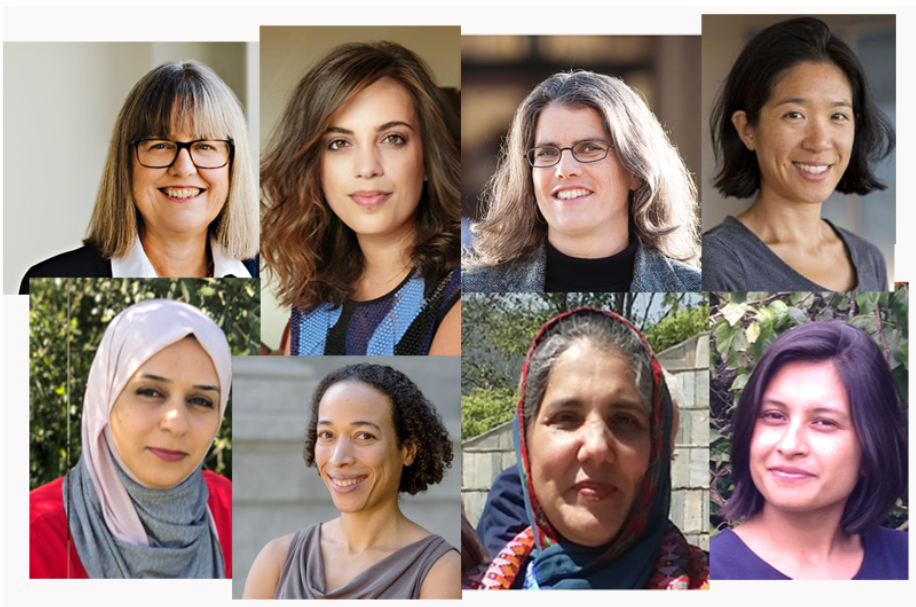

provided compelling arguments that a computer had finally achieved quantum "primacy" - the ability to outperform the best possible classical computer in a given task (see Viewpoint: Quantum Leap for Quantum Primacy). For this demonstration, USTC researchers used two different quantum computers, one based on superconducting circuits and one on photonic interferometry, solving "sampling" problems that would be hard to tackle classically. For both computer types, the researchers observed exceptional quantum computational speedups that made the claim of quantum primacy hard to dispute.

\section{More Women Physicists}

Since the dawn of physics research, women have unfairly held

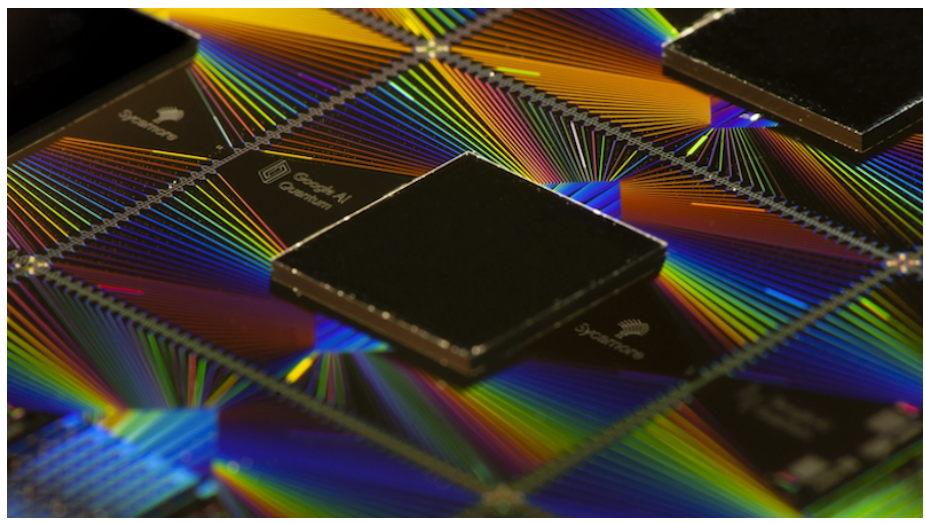

Credit: Google

the short end of the physics stick. This year, Physics paid attention to both what's improving and what still needs to change. Women physicists are receiving some of the highest honors in the field and are using their voices to promote the role of science in society (see Q\&A: The Women Who Win). Encouraging lessons on how to improve women's participation are coming from some Middle Eastern and Asian countries where women make up the majority of STEM graduate students (see Viewpoint: Why More Women Study Physics in Muslim Countries and Q\&A: Where Women Scientists Are the Majority). And promising emerging approaches to promoting gender balance involve creating an inclusive community that doesn't force women to change (see News Feature: Fixing a Physics Culture Problem).

\section{Green Materials Get Smart}

Solving climate change is a challenge that requires all forms of brain power. This year saw materials scientists enlisting artificial intelligence in the development of new materials for batteries, catalysts, and other green solutions (see News Feature: Al Promises Climate-Friendly Materials). Machine-learning algorithms, for example, are sifting through large chemistry datasets, searching for relationships that human chemists might miss. These efforts have uncovered new materials, such as organic compounds that could improve battery lifetimes. Complementing these algorithms are autonomous labs, in which robots quickly synthesize and test chemical candidates. Researchers in the field expect that Al methods could reduce the time for material development by a factor of 10 . 


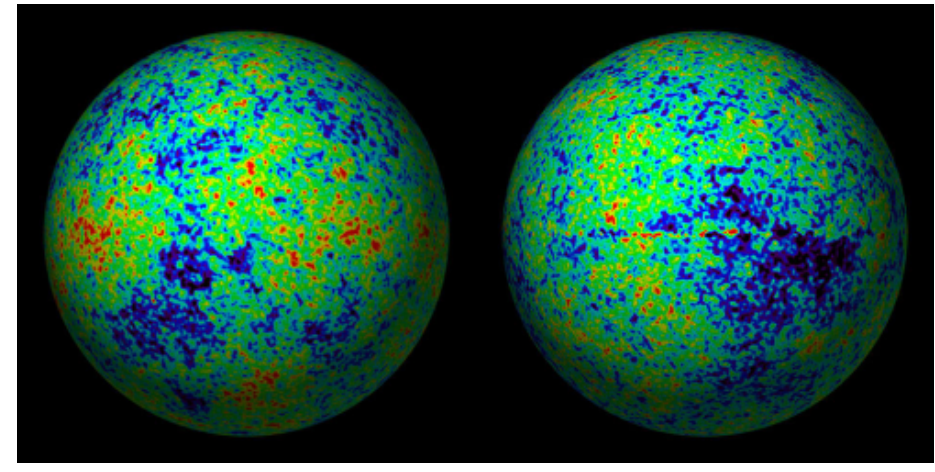

Credit: WMAP Science Team/NASA

\section{Google's Quantum Computer Turned Time Crystal}

Today's quantum computers are mostly limited to simple calculations, but researchers showed that Google's Sycamore quantum computer can perform another kind of trick: simulating a time crystal-a quantum system that evolves perpetually in periodic cycles (see Focus: Turning a Quantum Computer into a Time Crystal). But here's the weird part: on a quantum computer, the distinction between simulation and demonstration of a time crystal is blurred. Classical computers can only simulate a time crystal's behavior. But the many-body quantum system that makes up the researchers' device behaves as an actual time crystal. So, quantum computers can do more than calculate!

\section{Climate Change Fingerprints}

This year, heat waves, floods, and hurricanes left a sobering impact on the planet. Climate models say these events will occur more often and with increasing severity as Earth's overall temperatures rise. But attributing a specific extreme weather event to climate change is tricky. Researchers developed a new statistical method that compares modeled patterns of extreme events to observed patterns, offering a tool for establishing a correlation between extreme weather events and climate change (see Research News: Pinpointing the Roots of Extreme Weather Events). Testing the model with past data, the team concluded that two heat waves that occurred in Asia in 2010 were likely a manifestation of the climate's natural variability. But they suggest that a disproportionately large heat wave that affected Canada in 2021 could be linked to climate change.

\section{Alternative Dark Matter Explanation Scores a Point}

While searches for dark matter continue to come up empty, some researchers are pursuing an alternative approach that assumes that dark matter doesn't exist and that, instead, gravity is more complicated than presently thought. Predictions from this so-called MOND (modified Newtonian dynamics) theory agree with many astronomical observations but not with some observations of the cosmic microwave background (CMB). Now, theorists have made progress on fixing this issue by proposing a version of the theory that fits CMB data (see Focus: Dark Matter Alternative Passes Big Test). The updated model increases MOND's credibility, but as with previous versions, the theory postulates new fields that lack a theoretical motivation, so most cosmologists remain unconvinced.

\section{Neutron Stars as Cosmic Labs}

Short of a black hole, no astrophysical body is denser than a neutron star. Their extreme gravitational pull thus makes neutron stars ideal environments for "strong-field" tests of the theory of general relativity. Such tests, however, require better knowledge of the "equation of state" of ultradense matter. This year, researchers circumvented this problem by describing stellar properties with universal relations that are independent of such an equation. They then constrained those properties by combining $x$-ray observations of a neutron star with gravitational-wave detections of a merging neutron star pair. This "multimessenger" approach allowed them to put new limits on how gravity may violate certain types of symmetries (see Viewpoint: Compiling Messages from Neutron Stars).

\section{A Playful Spin on Physics}

In other rotating-body news, this year saw a new, star-inspired spinning top arrive on the market. Now retired from research, astrophysicist Kenneth Brecher designs tops with intriguing behaviors that incorporate mathematical constants into their geometry. In his latest creation, dubbed the DeltaCELT, the ratio of the top's long and short axes equals the Feigenbaum constant, which determines when certain systems become chaotic. DeltaCELT is a rattleback-a top with a preferred spinning direction. If spun clockwise, it slows to a stop and, with a rattling motion, starts spinning the other way (see Arts \& Culture: One-Way Spinning Top). Brecher presented the top at the Bridges conference, a meeting dedicated to mathematics-inspired art (see Research News: Physics Abounds at Conference on Math and Art). 


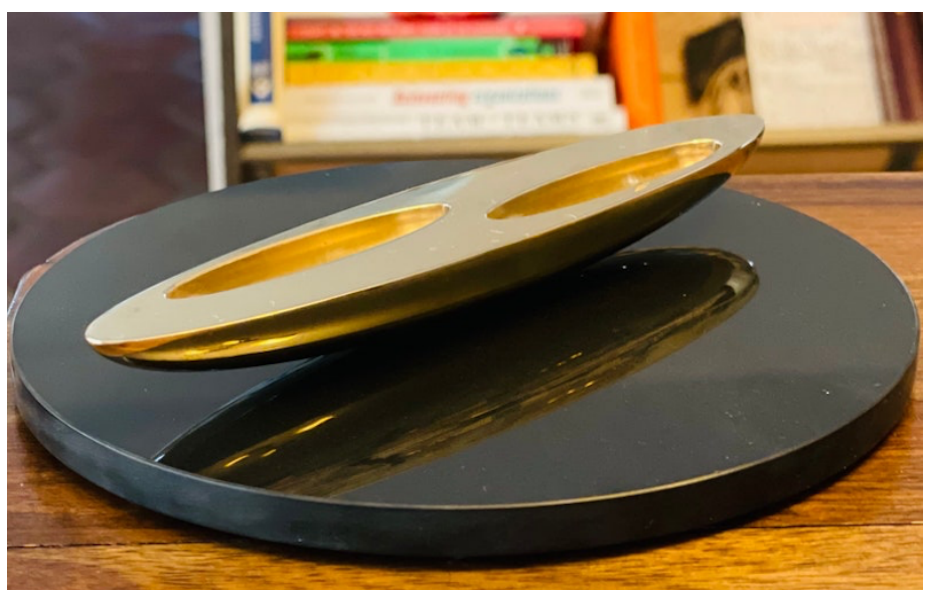

Credit: Karen Brecher 